International Journal of Medical Sciences ISSN 1449-1907 www.medsci.org 2006 3(1):21-25 Research paper

\title{
Maternal use of Loratadine during pregnancy and risk of hypospadias in offspring
}

\author{
Lars Pedersen, Mette Vinther Skriver, Mette Nørgaard, Henrik Toft Sørensen \\ Department of Clinical Epidemiology, Aarhus University Hospital, Ole Worms Alle 1150, DK-8000 Aarhus C, Denmark
}

Corresponding address: Lars Pedersen, Department of Clinical Epidemiology, Ole Worms Alle 1150, DK-8000 Aarhus C, Denmark. Tel: + 458942 4805, Fax: + 458942 4801, E-mail: lap@,dce.au.dk

Received: 2005.11.25; Accepted: 2006.01.05; Published: 2006.01.31

To examine the risk of hypospadias after exposure to loratadine and other antihistamines during pregnancy, we conducted a population-based case-control study in four Danish counties, which account for $30 \%$ of the Danish population $(\sim 1.6 \mathrm{M})$. We obtained data on maternal use of antihistamines from prescription databases, and data on birth outcomes from the Danish Medical Birth Registry (MBR) and the Hospital Discharge Registry (HDR). A total of 65,383 male births with a full prescription history of the mother in the study period from 1989-2002 were available for analysis. Within this cohort, we identified cases with a diagnosis of hypospadias, and 10 selected controls per case without such a diagnosis (matched on birth month, gender and year of birth). We identified 227 cases of hypospadias recorded in the HDR within six months postpartum and 2270 controls. One case $(0.4 \%)$ and eight $(0.4 \%)$ controls were exposed to loratadine in the first trimester and up to 30 days before the time of conception. The adjusted odds ratio (OR) for hypospadias among users of loratadine relative to non-users was 1.4 (95\% CI: 0.2-11.2) and the corresponding OR for other antihistamines was 1.9 (95\% CI: 0.7-5.7). In this study, maternal exposure to loratadine did not appear to be associated with an increased risk of hypospadias when compared with other antihistamines, although it should be noted that the statistical precision of the risk estimates might be limited.

Key words: Hypospadias, Loratadine, pregnancy, drug safety, case-control studies

\section{Introduction}

Hypospadias occurs with a reported prevalence of $0.3 \%$ to $0.8 \%$ and since the $1970 \mathrm{~s}$, multiple reports from the United States, England, Scandinavia, and Hungary have shown an increase in the occurrence of hypospadias [1-7]. Although very few risk factors for hypospadias are established, gestational and preexisting diabetes, intrauterine growth retardation, paternal subfertility, in vitro fertilization (IVF), maternal age, and genetic factors have all been suggested to be associated with an increased risk of hypospadias [8-17].

Loratadine is a non-sedating antihistamine commonly used for seasonal allergies [18]. In 2001, a report from Sweden suggested an association between maternal use of loratadine and infant hypospadias [19]. Having considered year of birth, maternal age, and parity, the odds ratio (OR) for hypospadias in relation to loratadine exposure was 2.39 (95\% confidence interval [CI]: 1.43-3.38). The study also compared the occurrence of hypospadias after the use of other antihistamines. The OR for having a diagnosis of hypospadias in the Swedish Medical Birth Registry (MBR) after maternal use of loratadine compared with maternal use of other antiallergic anti-histamines was 4.0 (95\% CI:1.42-12.9) [19].

Neither a recent study from Israel including 210 pregnant women exposed to loratadine [20] nor a study that used data from four countries and included 161 pregnant women exposed to loratadine [21] found an increased risk of hypospadias. However, these studies had limited power and due to the low prevalence of hypospadias not one case could be expected. Recently, the American Centers of
Disease Control and Prevention (CDC) reported a case-control study including 563 infants with secondor third-degree hypospadias [22]. This study did not find any association between loratadine use and hypospadias. But since first-degrees hypospadias was excluded CDC could not assess the potential association between the mildest form of hypospadias and loratadine. Since use of anti-allergic drugs is common, any causal association may have major public health implications. We, therefore, conducted a case-control study in Denmark based on hospital discharge data of cases with hypospadias and population controls linked to Danish prescription registries.

\section{Materials and methods}

\section{Study population}

The study was conducted in the four Danish counties of North Jutland, Aarhus, Viborg and Ringkoebing which account for $30 \%$ of the Danish population $(\sim 1.6 \mathrm{M})$. A total of 65,383 male births with a full prescription history of the mother were available for analyses in the study period from 19892002 (North Jutland $n=34,859$ ), 1996-2002 (Aarhus $\mathrm{n}=20,382$ ) and 1998-2002 (Viborg $\mathrm{n}=4,148$ ) and (Ringkoebing $\mathrm{n}=5,994)$.

\section{Cases of hypospadias}

We identified all cases of hypospadias in the period 1989-2003 from the nationwide Hospital Discharge Registry (HDR). This Registry comprises of data on all discharges from hospitals in Denmark and includes 10-digit personal identifiers, dates of admission and discharge, surgical procedures, and up to 20 diagnoses [23] classified according to the Danish versions of the International Classification of 
Diseases, $8^{\text {th }}$ Revision (ICD-8) until the end of 1993 and ICD-10 thereafter (ICD-9 was never used in Denmark). The codes for hypospadias in ICD-8 are 752.20 (hypospadia glandis, $n=3$ ), 752.21 (hypospadia corporis penis, $\mathrm{n}=1$ ), 752.22 (hypospadia scrotalis, $n=0), 752.28$ (hypospadia alia definite, $n=0$ ), 752.29 (hypospadia, $n=5)$; in ICD-10, the codes are Q54.0 (hypospadia glandis, $\mathrm{n}=101$ ), Q54.1 (hypospadia corporis penis, $\mathrm{n}=11$ ), Q54.2 (hypospadia penoscrotalis, $\mathrm{n}=0$ ), Q54.3 (hypospadia perinealis, $\mathrm{n}=2$ ), Q54.4 (hypospadia penis arcuatos, $\mathrm{n}=3$ ), Q54.8 (other specified hypospadias, $n=0$ ), Q54.9 (hypospadias without any specifications, $n=135$ ); There were 159 children with multiple hypospadias codes, and 25 children with both ICD-8 and ICD-10 codes. Using these codes, a total of 319 cases of hypospadias were identified (anytime postpartum) in the cohort of 65,383 male births in the four counties.

\section{The Danish Medical Birth Registry}

The MBR, which comprises of data collected by midwives and doctors attending deliveries, contains information on all births in Denmark since 1 January 1973 [24,25]. The main data constitute maternal age, self-reported smoking status at first antenatal visit, birth order, stillbirth, Apgar score, gestational age, height and weight of the neonate, and personal identifiers for both mother and child [24].

Use of loratadine, other antihistamines, IVF drugs,

antidiabetics and epileptics

As a part of the tax-funded healthcare for all inhabitants, the Danish National Health Service reimburses part of the patient expenditure on a wide range of prescribed drugs [21,26]. Danish patients are served by pharmacies equipped with electronic accounting systems that are used primarily to secure reimbursement for the National Health Service in each county. These systems include information on WHO's Anatomical Therapeutic Chemical (ATC) classification code, the amount of the drug prescribed, the personal identification number, and the date of drug dispension. Since January 11989 all data from North Jutland County have been stored in a prescription database maintained by the Department of Clinical Epidemiology, Aarhus University Hospital and since 2000 the Department of Clinical Epidemiology has also maintained similar research prescription databases from the three other counties. The data from these three counties are available from January 1, 1996 (Aarhus County) and January 1, 1998 (Ringkoebing and Viborg counties). Drugs sold over the counter are not available in these Prescriptions databases

Among cases and controls, prescriptions on loratadine (ATC codes: R06AX13), other antihistamines (ATC code: R06, except R06AX13), clomifene (ATC code: G03GB02), antidiabetics (ATC code: A10) and epileptics (ATC code: N03) was obtained from the prescription databases.

\section{Data on preeclampsia}

From the HDR we also obtained information on preeclampsia (ICD-8 codes: 637.03, 637.04, 637.09, 637.19; ICD-10 codes: 014, 015), since this has been found to be associated with hypospadias. The unique personal identifiers (CPR-numbers) were used to link records from all registries.

\section{Statistical analysis}

The association between use of loratadine and hypospadias was studied in a nested case-control design within the cohort of women who had a livebirth or a stillbirth after the 28th week of gestation. Use of loratadine was classified into three groups according to the time of exposure. The first trimester is considered the critical period for organ formation. Thus, the primary focus was the "early pregnancy" group, comprising of women who filled a prescription within 30 days before conception ("conception" was defined as the first day of last menstrual period [LMP]) up to the end of the first trimester (week 14 after the LMP). A second group comprised of women who filled a prescription within the first six months of pregnancy. A third group, the "entire pregnancy" group, comprised of women who filled prescriptions for loratadine at any time during pregnancy. Users of other antihistamines were classified similarly.

We restricted the first analysis to the pregnancies where the women lived in the four counties during the complete study period, which was the period between 30 days before conception and six months post-delivery. In the first analysis, cases were defined as boys with hypospadias recorded in the HDR during the first six months post-delivery.

The controls were selected from the study population of 65,383 male births. The control group comprised of 10 controls per case, and these controls had no recorded diagnosis of hypospadias during the first six months post delivery. We matched on birth, month, and year of the child. To examine whether the restriction of the hypospadias diagnosis to six months post-delivery had any impact on the results, we conducted a second analysis in which we defined cases as boys with hypospadias recorded in the HDR any time post-delivery (some children might have been coded later e.g. at the time of surgery) and controls as boys with no recorded diagnosis of hypospadias during the study period. In this analysis, cases and controls had to have lived in the four counties until the cases were diagnosed.

For the main study variables, we constructed contingency tables between exposure to loratadine, other antihistamines, case/control status and possible confounders. We used exact conditional logistic regression to estimate the relative risk by virtue of the OR of hypospadias associated with exposure to loratadine adjusted for maternal age, birth order, smoking status, preeclampsia, use of clomifene (a proxy for IVF), diabetes, and epilepsy. The analyses were done using SAS version 9.1 (SAS Inc., Cary, NC, USA).

\section{Results}

We identified 227 cases of hypospadias and 2270 matched controls when considering diagnosis within six months postpartum. Descriptive data for cases and controls are shown in Table 1. A total of one case and eight controls were exposed to loratadine in the first trimester or up to 30 days before the time of conception compared with four cases and 23 controls 
exposed to other antihistamines in the first trimester or up to 30 days before the time of conception.

Table 2 shows the ORs for hypospadias associated with exposure to loratadine and other antihistamines according to the time of exposure. The adjusted OR for loratadine exposure within 30 days before conception and during the first trimester was 1.4 (95\% CI: 0.0-10.5). The adjusted OR for other antihistamines was 1.9 (95\% CI: 0.5-5.8). The crude and adjusted odds ratios were similar, suggesting that the variables we controlled for were no major confounders.

For the second group, who filled the prescription within the first six month of pregnancy, and the third, "entire pregnancy" group, the adjusted ORs for loratadine exposure were 0.8 (95\% CI: $0.0-4.9)$ and 0.5 (95\% CI: 0.0-3.3), respectively. The adjusted ORs for other antihistamines were 1.6 (95\% CI: 0.3-5.5) and 1.0 (95\% CI: 0.2-3.4), respectively.

Table 1. Characteristics of 227 cases of hypospadias recorded within six months postpartum and 2270 control subjects.

\begin{tabular}{|c|c|c|}
\hline Variable & $\begin{array}{c}\text { Cases } \\
N(\%)\end{array}$ & $\begin{array}{c}\text { Controls } \\
N(\%)\end{array}$ \\
\hline Exposure to Loratadine* & $1(0.4)$ & $22(1.0)$ \\
\hline 30 days before conception and first trimester & $1(0.4)$ & $8(0.4)$ \\
\hline First trimester and second trimester & $1(0.4)$ & $15(0.7)$ \\
\hline During pregnancy & $1(0.4)$ & $21(0.9)$ \\
\hline Exposure to other antihistamines* & $4(1.8)$ & $40(1.8)$ \\
\hline 30 days before conception and first trimester & $4(1.8)$ & $23(1.0)$ \\
\hline First trimester and second trimester & $3(1.3)$ & $21(0.9)$ \\
\hline During pregnancy & $3(1.3)$ & $30(1.3)$ \\
\hline Maternal age & & \\
\hline$<25$ & $41(18.1)$ & $319(14.1)$ \\
\hline $25-30$ & $99(43.6)$ & $1,036(45.6)$ \\
\hline$>30$ & $87(38.3)$ & $915(40.3)$ \\
\hline Birth order & & \\
\hline 1 & $108(47.6)$ & $942(41.5)$ \\
\hline $1+$ & $119(52.4)$ & $1,328(58.5)$ \\
\hline Smoking 1991-2002 & & \\
\hline Yes & $51(22.5)$ & $524(23.1)$ \\
\hline No & $156(68.7)$ & $1,571(69.2)$ \\
\hline Missing & $20(8.8)$ & $175(7.7)$ \\
\hline Gestational age & \multicolumn{2}{|c|}{} \\
\hline$\geq 37$ weeks & $198(87.2)$ & $2,160(95.2)$ \\
\hline $34-36$ weeks & $20(8.8)$ & $81(3.6)$ \\
\hline$<34$ weeks & $9(4.0)$ & $29(1.3)$ \\
\hline Prescription for ovulation-inducing drugs & $1(0.4)$ & $44(1.9)$ \\
\hline Maternal epilepsy & $2(0.9)$ & $13(0.6)$ \\
\hline Maternal diabetes & $1(0.4)$ & $8(0.4)$ \\
\hline Preeclampsia & $13(5.7)$ & $48(2.1)$ \\
\hline
\end{tabular}

*Exposure during pregnancy and 30 days before conception

Considering all cases of hypospadias recorded anytime post-delivery $(N=319)$, the risk estimates did not change markedly. The adjusted OR for exposure to loratadine in the first trimester and 30 days before conception was 1.1 (95\% CI: 0.0-7.7), and the OR for exposure to other antihistamines in the same period was 1.7 (95\% CI: 0.5-4.7). The adjusted OR for exposure to loratadine within the first six months of pregnancy was 0.6 (95\% CI 0.0-3.8) and for the entire pregnancy 0.5 (95\% CI 0.0-2.7). The adjusted ORs for other antihistamines were 1.1 (95\% CI: 0.2-3.7) and 0.7 (95\% CI: 0.1-2.3), respectively. The risk point estimates were generally higher for other antihistamines than for loratadine.
Since we only had one exposed case, our dataset did not allow separate analyses of hypospadias as a single outcome or as an outcome in combination with other congenital malformations. Such an analysis might have been useful in order to examine the presence of surveillance bias, as hypospadias occur in clusters with other malformations in some children.

\section{Discussion}

The current study has shown that maternal exposure to loratadine does not appear to be associated with an increased risk of hypospadias compared with other antihistamines. In fact, the risk point estimates for hypospadias were higher with maternal exposure to other antihistamines compared with loratadine. Thus, our risk estimates do not corroborate the findings in the Swedish study [19] that initiated the hypospadias debate. However our risk estimates had limited statistical precision and an effect similar to that in the Swedish study cannot be ruled out entirely.

Table 2. The association between hypospadias recorded within six months postpartum and maternal use of antihistamines according to time of exposure, odds ratios (OR) and 95\% confidence intervals $(\mathrm{CI})$

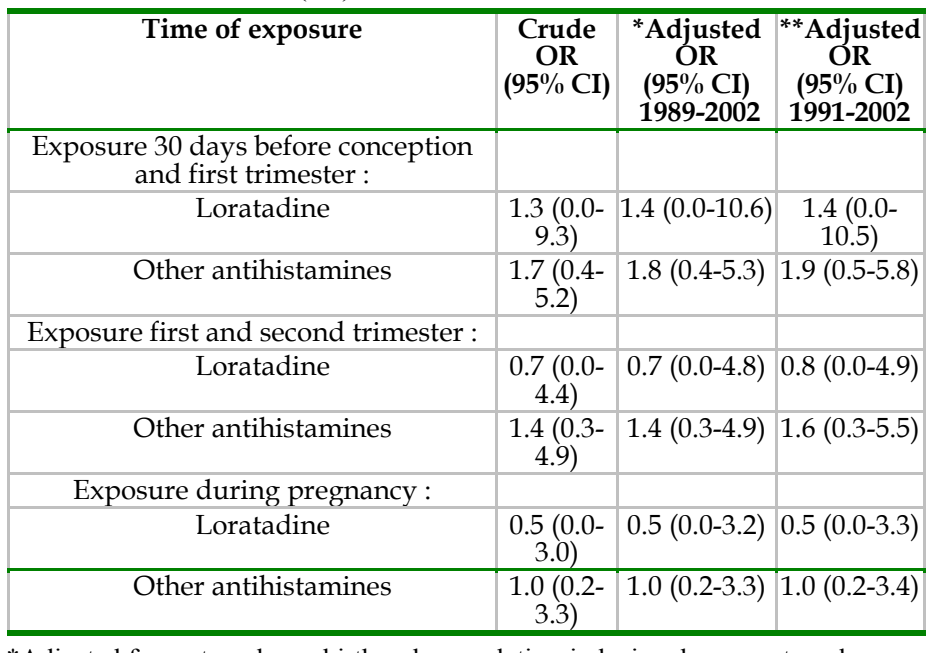

*Adjusted for maternal age, birth order, ovulation-inducing drugs, maternal epilepsy, maternal diabetes and preeclampsia.

**Adjusted for smoking, maternal age, birth order, ovulation-inducing drugs, maternal epilepsy, maternal diabetes and preeclampsia.

Our case-control study had complete and independent registration of birth, birth outcome, and prescription data which prevented selection bias and some types of information bias; since the study was based on routinely recorded data, independent of the diagnosis. Importantly, there was no risk of recall bias, which can invalidate case-control studies that solely rely on interviews [27]. Although smaller than the Swedish Birth Registry, the database we used is one of the largest in the world for studying the safety of drugs used in pregnancy and previous studies have shown high data quality in both the prescription database and the Birth Registry $[25,28]$. Coding errors occur in less than 0.5 percent of cases in the prescription database [28].

Our study was based on the HDR, and it is known that discharge diagnoses listed in discharge registries are not always accurate. We reviewed 43 records of the hypospadias cases in our study and 
found only three to be misclassified. Generally, lack of specificity, biases risk estimates towards unity. However, our prevalence of hypospadias corresponds to the prevalence reported in other datasets.

Loratadine is also sold "over the counter" in Denmark and since the prescription databases do not capture information regarding "over the counter" medication, the exposure information may be incomplete. Incomplete exposure information in the current study may bias the results towards unity as well.

Because of our reliance on dispensing information in the record linkage study, we do not know whether the women in the study actually took the drugs. However, the fact that patients are required to pay partially for the costs themselves is likely to have improved compliance.

We were able to adjust for possible confounding factors except for the years 1989 and 1990, where we did not have information regarding smoking. However, in our study, adjustment for the available confounding variables did not change the unadjusted risk estimates substantially, implying that these variables were no major confounders. Since the development of the external organs is initiated in the early fetal period, some of the studied variables such as preeclampsia should be interpreted as biological characterization of infants born with hypospadias rather than possible causal factors.

Our data are in line with the few other existing studies. Thus, in a recently conducted study by the CDC no association between maternal use of loratadine and second- or third-degree hypospadias was demonstrated [22]. Similarly, in an Israeli study [20], no increased risk of hypospadias was demonstrated in the loratadine group compared with other antihistamines. Moretti and coworkers found in a multi center study [21] that maternal exposure to loratadine was not associated with major malformations.

However, the infrequent maternal use of loratadine and the prevalence of hypospadias have a major impact on sample size requirements for providing the definitive assurances of the safety of loratadine to the unborn child [29]. Thus, to rule out a doubling of the risk of hypospadias would, based on our registries, require a study with 1,350 cases of hypospadias and 13,500 controls (power 80 percent and $0.5 \%$ exposure prevalence among controls).

\section{Conclusion}

In conclusion, maternal exposure to loratadine does not appear to be associated with an increased risk of hypospadias compared to other antihistamines. However, the statistical precision of our risk estimates was limited.

\section{Conflict of interest}

See Acknowledgements.

\section{Acknowledgements}

The study was supported by an unrestricted grant from the Schering-Plough Research Institute, New Jersey, USA, Apotekerfonden af 1991 (The Danish Pharmaceutical Association) and C.W. Obels Fond (The C.W. Obel Foundation).

\section{References}

1. Matlai P, Beral V. Trends in congenital malformations of external genitalis. Lancet 1985;1:108

2. Bjerkedal T, Bakketeig LS. Surveillance of congenital malformations and other conditions of the newborn. Int J Epidemiol 1975;4:31-6.

3. Kallen B, Winberg J. An epidemiologic study of hypospadias in Sweden. Acta Paediatr Scand 1982;293:1-21.

4. Silver RI, Rodriguez R, Chang TS, Gearhart JP. In vitro fertilization is associated with an increased risk of hypospadias. J Urol 1999;161:1954-7.

5. Czeizel A. Increasing trends in congenital malformations of male external genitalia. Lancet 1985;1:462-3.

6. Paulozzi LJ, Erickson D, Jackson RJ. Hypospadias in trends in two US surveillance systems. Pediatrics 1997;100:831-4.

7. Gallentine ML, Morey AF, Thompson I. Hypospadias: a contemporary epidemiologic assessment. Urology 2001;57:788-90.

8. Åberg A, Westbom L, Källén B. Congenital malformations among infants whose mothers had gestational diabetes or preexisting diabetes. Early Hum Develop 2001; 61:85-95.

9. Fredell L, Kockum I, Hansson E, Holmner S, Lundquist L, Läckgren $\mathrm{G}$, et al. Heredity of hypospadias and the significance of low birth weight. J Urol 2002; 167:1423-7.

10. Hussain N, Chaghtai A, Herndon CD, Herson VC, Rosenkrantz TS, McKenna PH. Hypospadias and early gestation growth restriction in infants. Pediatrics 2002;109: 473-8.

11. Wu WH, Chuang JH, Ting YC, Lee SY, Hsieh CS. Developmental anomalies and disabilities associated with hypospadias. J Urol 2002;168:229-32.

12. Silver RI. What is the etiology of hypospadias? A review of recent research. Del Med J 2000;72:343-7.

13. Fredell L, Lichtenstein P, Pedersen NL, Svensson J, Nordenskjold A. Hypospadias is related to birth weight in discordant monozygotic twins. J Urol 1998;160:2197-9.

14. Weidner IS, Møller H, Jensen TK, Skakkebæk NE. Risk factors for cryptorchidism and hypospadias. J Urol 1999;161:1606-9.

15. Chambers EL, Malone PS. The incidence of hypospadias in two English cities: a case-control comparison of possible causal factors. BJU International 1999;84:95-8.

16. Wennerholm UB, Bergh C, Hamberger L, Lundin K, Nilsson L, Wikland $\mathrm{M}$, et al. Incidence of congenital malformations in children born after ICSI. Hum Reprod 2000; 15:944-8.

17. Klip H, Verloop J, van Gool JD, Koster ME, Burger CW, van Leeuwen FE; OMEGA Project Group.. Hypospadias in sons of women exposed to diethylstilbestrol in utero: a cohort study. Lancet 2002;359:1102-7.

18. Dansk Lægemiddel information. Lægemiddelkataloget 2004 Copenhagen, Denmark: Dansk Lægemiddel Information. 2004.

19. Källén B, Olausson PO. Monitoring of maternal drug use and infant congenital malformations. Does loratadine cause hypospadias? Int J Risk Safety Med 2002; 14:115-9.

20. Diav-Citrin O, Shechtman S, Aharonovich A, Moerman L, Arnon J, Wajnberg R, et al. Pregnancy outcome after gestational exposure to loratadine or antihistamines: a prospective controlled cohort study. J Allergy Clin Immunol 2003;111:1239-43.

21. Moretti ME, Caprara D, Coutinho CJ, Bar-Oz B, Berkovitch M, Addis A, Jovanovski E, Schuler-Faccini L, Koren G. Fetal safety of loratadine use in the first trimester of pregnancy: a multicenter study. J Allergy Clin Immunol 2003;111:479-83.

22. Centers for Disease Control and Prevention (CDC). Evaluation of an association between loratadine and hypospadias--United States, 1997-2001. MMWR Morb Mortal Wkly Rep. 2004;53:219-21.

23. Andersen TF, Madsen M, Jørgensen J, Mellemkjær L, Olsen JH. The Danish National Hospital Register. A valuable source of data for modern health sciences. Dan Med Bull 1999;46:263-8.

24. Knudsen LB, Olsen J. The Danish Medical Birth Registry. Dan Med Bull 1998; 45:320-3.

25. Kristensen J, Langhoff-Roos J, Skovgaard LT, Kristensen FB. Validation of the Danish Birth Registration. J Clin Epidemiol 1996;49:893-7.

26. Gaist D, Sørensen HT, Hallas J. The Danish prescription registries. Dan Med Bull 1997;44:445-8. 
27. Olsen J, Czeizel A, Sørensen HT, Nielsen GL, de Jong van den Berg LT, Irgens LM, et al. How do we best detect toxic effects of drugs taken during pregnancy? A EuroMap paper. Drug Saf 2002;25:21-32.

28. Olsen JH, Sørensen HT, Friis S, McLaughlin JK, Steffensen FH, Nielsen GL, et al. Cancer risk in users of calcium channel blockers. Hypertension 1997;29:1091-4.

29. Mitchel AA. Special considerations in studies of drug-induced birth defects. In: Strom BL, Ed. Pharmacoepidemiology. Chichester: John Wiley \& Sons. 1994: 595-608. 